1 Hacettepe Journal of Mathematics and Statistics

h Volume 47 (1) (2018), $101-106$

\title{
Some results on mean open, closed and clopen sets
}

\author{
Ajoy Mukharjee ${ }^{* \dagger}$ and Kallol Bhandhu Bagchi ${ }^{\ddagger}$
}

\begin{abstract}
In this paper, we obtain some more properties of mean open and closed sets. We also introduce a notion of mean clopen sets and obtain some properties of such sets. We observe that a mean clopen set is both mean open and mean closed but the converse is not true.
\end{abstract}

Keywords: Maximal open set, Minimal open set, Mean open set, Mean closed set, Mean clopen set.

2000 AMS Classification: 54A05, 54C05.

Received: 11.01.2017 Accepted: 28.02.2017 Doi : 10.15672/HJMS.2017.447

\section{Introduction}

Unless otherwise mentioned, $X$ stands for the topological space $(X, \mathscr{P})$. By a proper open set (resp. closed set) of a topological space $X$, we mean an open set $G \neq \emptyset, X$ (resp. a closed set $E \neq \emptyset, X)$.

In the literature, we find a considerable number of articles on minimal and maximal open and closed sets due to Nakaoka and Oda $[4,5,6]$. A nonempty open set $U$ of $X$ is said to be a minimal open set [6] if $\emptyset$ and $U$ are only open sets contained in $U$. Dualizing the notion of minimal open set, Nakaoka and Oda [5] introduced the notion of maximal open sets: a proper open set $U$ of $X$ is said to be a maximal open set [5] if $U$ and $X$ are only open sets containing $U$. As consequences of minimal and maximal open sets, Nakaoka and Oda [4] also introduced and studied minimal closed sets (Definition 2.3) and maximal closed sets (Definition 2.5). We see that if $G$ is a minimal open set and $H$ is a maximal open set in $X$, then either $G \subset H$ or $X$ is disconnected [2, Theorem 2.6]. If $G \subset H$ for a minimal open set $G$ and a maximal open set $H$ of a topological space $X$, then there has a possibility to exist an open set $U$ such that $G \subset U \subset H$. Here $U$ lies between two open sets $G, H$ in the inclusion sense like the arithmetic mean of two real numbers which instigates us to introduce the notion of mean open sets (Definition

\footnotetext{
*Department of Mathematics, St. Joseph's College, Darjeeling, W. Bengal- 734 104, India, Email: ajoyjee@gmail.com

†Corresponding Author.

${ }^{\ddagger}$ Department of Mathematics, Kalipada Ghosh Tarai Mahavidyalaya, Bagdogra, Darjeeling, W. Bengal- 734 014, India, Email: kbagchi.789@gmail.com
} 
2.7). Focusing on clopen sets in the light of mean open and closed sets, we find some odd behaviors of clopen sets which inspire us to initiate the study of mean clopen sets (Definition 3.8). It is easy to see that mean clopen sets are both mean open and mean closed but the converse is not true (Example 3.9). We also observe that mean clopen sets appear in pairs (Theorem 3.11) which reveals a beauty of the notion of mean clopen sets.

Throughout the paper, $R$ stands for the set of real numbers. For a subset $A$ of a topological space $(X, \mathscr{P})$, we write $C l(A)$ (resp. Int $(A))$ to denote the closure (resp. interior) of $A$ with respect to the topological space $(X, \mathscr{P})$.

\section{Mean open and closed sets}

Firstly, we recall the following.

2.1. Theorem (Nakaoka and Oda [5]). If $U$ is a maximal open set and $W$ is an open set in $X$, then either $U \cup W=X$ or $W \subset U$. If $W$ is also a maximal open set distinct from $U$, then $U \cup W=X$

2.2. Theorem (Nakaoka and Oda [6]). If $U$ is a minimal open set and $W$ is an open set in $X$, then either $U \cap W=\emptyset$ or $U \subset W$. If $W$ is also a minimal open set distinct from $U$, then $U \cap W=\emptyset$.

2.3. Definition (Nakaoka and Oda [4]). A proper closed set $E$ of $X$ is said to be a minimal closed set if $\emptyset$ and $E$ are only closed sets contained in $E$.

2.4. Theorem (Nakaoka and Oda [4]). If E is a minimal closed set and $F$ is any closed set in $X$, then either $E \cap F=\emptyset$ or $E \subset F$. If $F$ is also a minimal closed set distinct from $E$, then $E \cap F=\emptyset$.

2.5. Definition (Nakaoka and Oda [4]). A proper closed set $E$ of $X$ is said to be a maximal closed set if $E$ and $X$ are only closed sets containing $E$.

2.6. Theorem (Nakaoka and Oda [4]). If $E$ is a maximal closed set and $F$ is any closed set in $X$, then either $E \cup F=X$ or $F \subset E$. If $F$ is also a maximal closed set distinct from $E$, then $E \cup F=X$.

The following two are duality principles due to Nakaoka and Oda [4]:

(i) $F$ is minimal closed in $X$ iff $X-F$ is maximal open in $X$.

(ii) $F$ is maximal closed in $X$ iff $X-F$ is minimal open in $X$.

2.7. Definition (Mukharjee and Bagchi [3]). An open set $M$ of a topological space $X$ is said to be a mean open set if there exist two distinct proper open sets $U, V(\neq M)$ such that $U \subset M \subset V$.

2.8. Definition (Mukharjee and Bagchi [3]). A closed set $E$ of a topological space $X$ is said to be a mean closed set if there exists two distinct proper closed sets $D, F(\neq E)$ such that $D \subset E \subset F$.

2.9. Theorem (Mukharjee and Bagchi [3]). An open set of a topological space is a mean open set iff its complement is a mean closed set.

2.10. Theorem. If $U$ and $V$ are two distinct minimal open sets in $X$, then $A=C l(A)$ or $X-C l(A)$ is a mean open set in $X$ where $A=U$ or $V$.

Proof. Since $U, V$ are distinct minimal open sets, we have by Theorem $2.2, U \cap V=\emptyset$ which implies that $U \cap C l(V)=\emptyset$. It means that $U \subset X-C l(V)$. Since $U, V$ are minimal open sets, $X-C l(V) \neq \emptyset, X$. A proper open set of a topological space if not a mean 
open set, either minimal open or maximal open. As $U \subset X-C l(V), X-C l(V)$ is not a minimal open set. Now if possible, let $X-C l(V)$ is a maximal open set. Since $V$ is a minimal open set, we have $V \subset X-C l(V)$ or $V \cup(X-C l(V))=X . V \subset X-C l(V)$ is not possible. From $V \cup(X-C l(V))=X$, we get $V=C l(V)$. If $V=C l(V)$ is not true, then $X-C l(V)$ is a mean open set in $X$. Proceeding similarly, we have either $U=C l(U)$ or $X-C l(U)$ is a mean open set.

If $V=C l(V)$, then $V$ is a clopen set and so the space is disconnected. Hence Theorem 2.10 may be stated as follows: If $U, V$ are two distinct minimal open sets, then the space is disconnected or $X-C l(U)$ and $X-C l(V)$ are mean open sets.

2.11. Corollary. If $E$ and $F$ are two distinct maximal closed sets in $X$, then $B=\operatorname{Int}(B)$ or $X-\operatorname{Int}(B)$ is a mean closed set in $X$ where $B=E$ or $F$.

Proof. As $E$ and $F$ are two distinct maximal closed sets, $X-E$ and $X-F$ are two distinct minimal open sets. By Theorem 2.10, $A=C l(A)$ or $X-C l(A)$ is a mean open set in $X$ where $A=X-E$ or $X-F$. For $A=X-E$, we get $E=\operatorname{Int}(E)$ from $A=C l(A)$ or from $X-C l(A)$, we get $X-C l(X-E)=\operatorname{Int}(E)$ is a mean open set. So $X-\operatorname{Int}(E)$ is a mean closed set by Theorem 2.9. Similarly, for $A=X-F$, we see that either $F=\operatorname{Int}(F)$ or $X-\operatorname{Int}(F)$ is a mean closed set by Theorem 2.9.

2.12. Theorem. Let $M$ be a maximal open set in a topological space $X$.

(i) If $\mathscr{C}_{m o}(X)$ is a collection of mean open sets such that $G \cup M \neq X$ for each $G \in$ $\mathscr{C}_{\text {mo }}(X)$, then $\bigcup_{G \in \mathscr{C}_{m o}(X)} G \neq X$.

(ii) If $\mathscr{C}_{m o}(X)$ is a collection of mean open sets such that $G \cup M=X$ for each $G \in$ $\mathscr{C}_{\text {mo }}(X)$, then $\bigcap_{G \in \mathscr{C}_{\text {mo }}(X)} G \neq \emptyset$.

Proof. (i) Since $G \cup M \neq X$ for each $G \in \mathscr{C}_{\text {mo }}(X)$, by Theorem 2.1, we get $G \subset M$ for each $G \in \mathscr{C}_{m o}(X)$ which in turn implies that $\bigcup_{G \in \mathscr{C}_{m o}(X)} G \subset M$. Since $M \neq X$, it follows that $\bigcup_{G \in \mathscr{C}_{m o}(X)} G \neq X$.

(ii) Since $G \cup M=X$ for each $G \in \mathscr{C}_{m o}(X)$, we have $X-M \subset G$ for each $G \in \mathscr{C}_{m o}(X)$. So we get $X-M \subset \bigcap_{G \in \mathscr{C}_{m o}(X)} G$. As $M$ is a maximal open set, $M \neq X$ and so $\bigcap_{G \in \mathscr{C}_{m o}(X)} G \neq \emptyset$.

2.13. Theorem. Let $M$ be a minimal open set in a topological space $X$.

(i) If $\mathscr{C}_{m o}(X)$ is a collection of mean open sets such that $G \cap M \neq \emptyset$ for each $G \in$ $\mathscr{C}_{\text {mo }}(X)$, then $\bigcap_{G \in \mathscr{C}_{m o}(X)} G \neq \emptyset$.

(ii) If $\mathscr{C}_{\text {mo }}(X)$ is a collection of mean open sets such that $G \cap M=\emptyset$ for each $G \in$ $\mathscr{C}_{\text {mo }}(X)$, then $\bigcup_{G \in \mathscr{C}_{m o}(X)} G \neq X$.

Proof. (i) Since $G \cap M \neq \emptyset$ for each $G \in \mathscr{C}_{\text {mo }}(X)$, by Theorem 2.2, we get $M \subset G$ for each $G \in \mathscr{C}_{m o}(X)$ which implies that $M \subset \bigcap_{G \in \mathscr{C}_{m o}(X)} G$. Since $M \neq \emptyset$, it follows that $\bigcap_{G \in \mathscr{C}_{m o}(X)} G \neq \emptyset$.

(ii) Since $G \cap M=\emptyset$ for each $G \in \mathscr{C}_{m o}(X)$, we have $G \subset X-M$ for each $G \in \mathscr{C}_{m o}(X)$. So we get $\bigcup_{G \in \mathscr{C}_{m o}(X)} G \subset X-M$. As $M$ is a minimal open set, $M \neq \emptyset$ and so $\bigcup_{G \in \mathscr{C}_{m o}(X)} G \neq X$.

Dualizing Theorems 2.12 and 2.13, we have Theorems 2.14 and 2.15, respectively. The proofs of these two theorems are omitted, as the proofs are similar to the proofs of their primal Theorems 2.12 and 2.13 , respectively.

2.14. Theorem. Let $E$ be a minimal closed set in a topological space $X$. 
(i) If $\mathscr{C}_{m c}(X)$ is a collection of mean closed sets such that $E \cap F \neq \emptyset$ for each $F \in$ $\mathscr{C}_{m c}(X)$, then $\bigcap_{F \in \mathscr{C}_{m c}(X)} F \neq \emptyset$.

(ii) If $\mathscr{C}_{m c}(X)$ is a collection of mean closed sets such that $E \cap F=\emptyset$ for each $F \in$ $\mathscr{C}_{m c}(X)$, then $\bigcup_{F \in \mathscr{C}_{m c}(X)} F \neq X$.

2.15. Theorem. Let $E$ be a maximal closed set in a topological space $X$.

(i) If $\mathscr{C}_{m c}(X)$ is a collection of mean closed sets such that $E \cup F \neq X$ for each $F \in$ $\mathscr{C}_{m c}(X)$, then $\bigcup_{F \in \mathscr{C}_{m c}(X)} F \neq X$.

(ii) If $\mathscr{C}_{m c}(X)$ is a collection of mean closed sets such that $E \cup F=X$ for each $F \in$ $\mathscr{C}_{m c}(X)$, then $\bigcap_{F \in \mathscr{C}_{m c}(X)} F \neq \emptyset$.

\section{Mean Clopen Sets}

Firstly, we recall that a set which is both open and closed in a topological space is called a clopen set. By a proper clopen set of a topological space, we mean a clopen set $A \neq \emptyset, X$.

3.1. Definition (Mukharjee [1]). A proper clopen set $A$ of a topological space $X$ is said to be minimal clopen if $B$ is a clopen set such that $B \subset A$, then $B=A$ or $B=\emptyset$.

3.2. Definition (Mukharjee [1]). A proper clopen set $A$ of a topological space $X$ is said to be maximal clopen if $B$ is a clopen set such that $A \subset B$, then $B=A$ or $B=X$.

3.3. Theorem (Mukharjee [1]). If $A$ is a minimal clopen set and $B$ is a clopen set in $X$, then $A \cap B=\emptyset$ or $A \subset B$. If $A$ and $B$ both are minimal clopen, then $A \cap B=\emptyset$.

3.4. Theorem (Mukharjee [1]). If $A$ is a maximal clopen set and $B$ is a clopen set in $X$, then $A \cup B=X$ or $B \subset A$. If $A$ and $B$ both are maximal clopen, then $A \cup B=X$.

3.5. Theorem (Mukharjee [1]). If $A$ is minimal clopen in a topological space $X$, then $X-A$ is maximal clopen in $X$ and conversely.

3.6. Theorem (Mukharjee [1]). If $A$ is minimal clopen and $B$ is maximal clopen in $X$, then either $A \subset B$ or $A=X-B$.

3.7. Theorem (Mukharjee [1]). If a topological space $X$ contains a set $A$ which is both maximal and minimal clopen, then (i) $A$ and $X-A$ are the only sets in the space which are both maximal and minimal clopen, and (ii) $A$ and $X-A$ are the only proper clopen sets in the space.

We now introduce the following.

3.8. Definition. A proper clopen set $A$ of a topological space $X$ is said to be a mean clopen set if there exist two proper clopen sets $C, D \neq A$ such that $C \subset A \subset D$.

A mean clopen set is neither maximal clopen nor minimal clopen. It is clear that a mean clopen set is both mean open and mean closed. However, a set which is both mean open and closed need not be mean clopen.

3.9. Example. For $a, b \in R$ with $a<b$, we consider the topology $\mathscr{T}=\{\emptyset, R,(-\infty$, $a),(a, \infty),(-\infty, b),[b, \infty),\{b\},(a, b), R-\{a\},(-\infty, a) \cup[b, \infty),(-\infty, a) \cup\{b\},(-\infty, a) \cup$ $(a, b),(-\infty, b],(a, b],(-\infty, a) \cup(a, b]\}$ on $R$ generated by the base $\{\emptyset,(-\infty, a),(a, \infty),(-\infty$, $b),[b, \infty),\{b\},(a, b)\}$. In the topological space $(R, \mathscr{T}),(-\infty, b)$ is both mean open and mean closed but it is not mean clopen.

3.10. Example. For $a, b \in R$ with $a<b$, let $\mathscr{T}$ be the topology on $R$ generated by the basis $\{\emptyset,\{a\},\{b\},(-\infty, a),(a, \infty),(-\infty, b),(b, \infty),(a, b)\}$. In the topological space $(R, \mathscr{T}),(-\infty, a]$ and $[b, \infty)$ are mean clopen sets. But the union of $(-\infty, a]$ and $[b, \infty)$ 
is not a mean clopen set in $R$. Also $(a, \infty)$ and $(-\infty, b)$ are mean clopen sets in $R$. But the intersection $(a, b)$ of the mean clopen sets $(a, \infty)$ and $(-\infty, b)$ is not a mean clopen set. These lead to conclude that the union and intersection of mean clopen sets may not be mean clopen sets again.

3.11. Theorem. If $A$ is a mean clopen set in $X$, then $X-A$ is also a mean clopen set in $X$.

Proof. Straightforward.

In view of Theorem 3.11, we conclude that mean clopen sets appear in pairs. Also if a topological space consists of a mean clopen set, then there exist at least six proper clopen sets in that space.

3.12. Theorem. If a topological space $X$ contains a set which is both maximal and minimal clopen, then $X$ contains no mean clopen set.

Proof. Follows by Theorem 3.7.

3.13. Theorem. If a topological space $X$ contains a mean clopen set $A$, then no minimal clopen set of $X$ has nonempty intersection with both $A$ and $X-A$.

Proof. We have nothing to prove if the topological space contains no minimal clopen set. So we assume that there exists a minimal clopen set $M$ in $X$. Since $M$ is minimal clopen in $X$, by Theorem 3.5, $X-M$ is maximal clopen in $X$. As $A$ is a mean clopen set, $A \neq M, X-M$. Also by Theorem 3.3, we have $M \subset A$ or $M \cap A=\emptyset$ and by Theorem 3.4, we have $A \subset X-M$ or $A \cup(X-M)=X$. Obviously, $M \subset A$ together with $A \subset X-M$ is not possible. The following cases may arise.

Case I: $M \cap A=\emptyset$ and $A \cup(X-M)=X$. Then we get $M \subset X-A$ and $X-(X-M)=$ $M \subset A$. Therefore $M \subset A \cap(X-A)=\emptyset$ which is not possible.

Case II: $M \subset A$ and $A \cup(X-M)=X$. From $A \cup(X-M)=X$, we get $X-(X-M)=$ $M \subset A$.

Case III: $A \subset X-M$ and $M \cap A=\emptyset$. From $M \cap A=\emptyset$, we get $A \subset X-M$.

So only feasible cases are $M \subset A$ or $A \subset X-M$. If $M \subset A$ and $A \subset X-M$ both hold, then $M \subset A \subset X-M$ which is impossible.

Now $A \subset X-M$ implies that $M \subset X-A$. If $M \subset A$, then $M \cap(X-A)=\emptyset$ and if $M \subset X-A$, then $M \cap A=\emptyset$.

3.14. Corollary. If $A$ is a mean clopen set and $N$ is a maximal clopen set in a topological space $X$, then one and only one of $A \cup N=X$ and $A \cup(X-N)=X$ hold.

Proof. $N$ being a maximal clopen set, by Theorem 3.5, $X-N$ is a minimal clopen set. By Theorem 3.13, one and only one of $(X-N) \cap A=\emptyset$ and $(X-N) \cap(X-A)=\emptyset$ hold. $(X-N) \cap A=\emptyset$ implies that $N \cup(X-A)=X .(X-N) \cap(X-A)=\emptyset$ implies that $N \cup A=X$

3.15. Theorem. If there are two distinct maximal clopen sets $A, B$ and a mean clopen set $M$ in a topological space $X$, then $A \cap B \neq \emptyset$.

Proof. Similar to that of Theorem 3.12 [3].

3.16. Theorem. If there are two distinct minimal clopen sets $A, B$ and a mean clopen set $M$ in a topological space $X$, then $A \cup B \neq X$.

Proof. By Theorem 3.5, $X-A, X-B$ are maximal clopen sets and so by Theorem 3.15, $(X-A) \cap(X-B) \neq \emptyset$ which in turn implies that $A \cup B \neq X$. 
Acknowledgement. The authors are grateful to the referee for careful reading the manuscript and for providing some valuable suggestions towards the improvement of clarity of the article.

\section{References}

[1] Mukharjee, A. On maximal and minimal clopen sets, An. Univ. Oradea Fasc. Mat. 23 (2), 127-130, 2016.

[2] Mukharjee, A. On maximal, minimal open and closed sets, Commun. Korean Math. Soc. 30 (3), 277-282, 2015.

[3] Mukharjee, A. and Bagchi, K.B. On mean open and closed sets, Kyungpook Math. J. 56 (4), 1259-1265, 2016.

[4] Nakaoka, F. and Oda, N. Minimal closed sets and maximal closed sets, Int. J. Math. Math. Sci. 2006, Article ID 18647, 8 pages.

[5] Nakaoka, F. and Oda, N. Some properties of maximal open sets, Int. J. Math. Math. Sci. (21), 1331-1340, 2003.

[6] Nakaoka, F. and Oda, N. Some applications of minimal open sets, Int. J. Math. Math. Sci. 27 (8), 471-476, 2001. 